\title{
A local presentation of systemic disease
}

\author{
FN Birrell, RJ D’Souza, K Harris
}

A 67-year-old woman presented with a three-month history of bilateral hearing loss, worse on the right, without nasal or aural discharge and no haemoptysis. Examination showed fluid levels bilaterally, with the appearance of a middle ear mass through the tympanic membrane on the right. Audiograms demonstrated mixed sensorimotor and conductive deafness. Urea and electrolytes and liver function were normal, full blood count showed anaemia ( $\mathrm{Hb} 8.4 \mathrm{gdl}$, mean corpuscular volume $76 \mathrm{fl}$ ). Computed tomography (CT) was performed (figure).

The patient underwent a number of out-patient investigations for her anaemia and was transfused before being referred to nephrology with rapidly progressive renal impairment (urea $33.6 \mathrm{mmol} / \mathrm{l}$, creatinine $864 \mu \mathrm{mol}$ ) and acidosis $\left(\mathrm{HCO}_{3}-18 \mathrm{mmol} / \mathrm{l}\right)$, but a normal potassium $(4.9 \mathrm{mmol} / \mathrm{l})$. Alkaline phosphatase was elevated at $425 \mathrm{IU} / 1$ (normal $40-130 \mathrm{IU} / \mathrm{l})$, but liver function tests were otherwise normal. Haemoglobin was $10 \mathrm{~g} / \mathrm{dl}$ with an mean corpuscular volume of $79 \mathrm{fl}$. Inflammatory markers were raised (plasma viscosity 2.28, C-reactive protein $291 \mathrm{mg} / \mathrm{l})$. Ward microscopy showed red cell casts in the urine and ultrasound showed normalsized kidneys with increased echogenicity. Chest X-ray showed patchy interstitial shadowing with a solitary 4-cm nodule in the left mid-zone.

Renal biopsy was performed which was diagnostic. Auto-antibodies, including all classes of antineutrophil cytoplasmic antibodies (ANCA) were negative.

Department of Rheumatology, Cannock Chase Hospital, Cannock, UK FN Birrell

Department of Nephrology, Leicester General Hospital, Leicester, UK RJ D'Souza K Harris

Correspondence to FN Birrell, Department of Rheumatology, Royal Shrewsbury Hospital North, Mytton Oak Road, Shrewsbury SY3 8XQ, UK

Accepted 27 September 1995

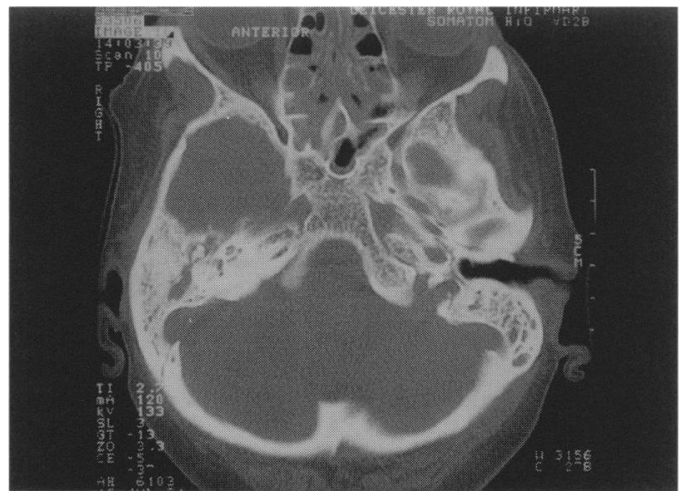

Figure CT scan of auditory apparatus

\section{Questions}

1 What is the diagnosis?

2 How would you treat this patient? 


\section{Answers}

\section{QUESTION 1}

Wegener's granulomatosis.

CT of the auditory apparatus showed an inflammatory mass disrupting the normal architecture of both media and interna otis. Such a mass could be caused by a cholesteatoma, but the bilateral auditory impairment and co-existent anaemia suggest a less localised problem.

Inflammatory markers can be a sensitive, if nonspecific guide to systemic illness. ANCA is often found in localised Wegener's granulomatosis, although less frequently than with generalised disease (see box 1). Urine stick testing remains a sensitive screening measure and abnormal specimens should be examined microscopically in this context. Inexpensive, noninvasive investigations such as chest $\mathrm{X}$-ray should not be forgotten.

Ferritin is an acute phase reactant, in addition to reflecting utilisable iron stores. Hence a low ferritin value, or normal value, in the face of an acute phase response confirms iron deficiency. Iron studies may assist in difficult cases. Iron deficient anaemia in this age group should probably be investigated for both upper and lower gut lesions, given the high incidence of dual pathology. In the absence of iron deficiency, a bone marrow aspirate plus trephine should be performed to exclude marrow infiltration.

Biopsy of the lesion is necessary if other screening investigations do not suggest a better site for biopsy (eg, transbronchial lung biopsy) and it is technically accessible. Biopsy may exclude cholesteatoma and guide early treatment. Cyclophosphamide plus prednisolone usually induces clinical remission of aural Wegener's granulomatosis. ${ }^{2}$ Although it might have prevented the development of renal failure in this patient, there is no controlled evidence favouring the use of potent immunosuppressive therapy in localised Wegener's granulomatosis.

Wegener's granulomatosis may be ANCAnegative at presentation; rarely, persistently so. This patient developed ANCA positivity at two weeks, in a perinuclear pattern of staining on immunofluorescence ( $p$-ANCA), at a titre of $1: 16$. In general, the more active and extensive the disease, the higher the ANCA positivity (and titre, where found). A cytoplasmic pattern of ANCA (c-ANCA) is particularly characteristic of Wegener's granulomatosis (box 2). The diagnosis rests on two from four of the 1990 American College of Rheumatology (ACR) criteria (box 3)

\section{QUESTION 2}

Oral prednisolone and cyclophosphamide is the treatment of choice. The role of adding in plasmapheresis is still controversial, but the

1 Nolle B, Specks U, Ludemann MS, Rohrbach MS DeRemee RA, Gross WL. Anticytoplasmic antibodies: their immunodiagnostic value in Wegener's granulomatosis. Ann Intern Med 1989; 111: 28-40.

2 Kempf H-G, Bootz F, Berg PA. Wegener's granulomatosis: otological and immunological aspects. Laryngorhinootologie otological and imm

\begin{tabular}{|ll|}
\hline $\begin{array}{l}\text { Sensitivity of ANCA and extent of } \\
\text { disease }\end{array}$ \\
\hline Disease & Sensitivity \\
generalised & $96 \%$ \\
loco-regional & $67 \%$ \\
remission & $32 \%$ \\
\hline
\end{tabular}

Box 1

Common patterns of ANCA staining

$c-A N C A$

- antigen is a $29 \mathrm{kDa}$ serine protease

- known as proteinase-3

- strong association with Wegener's granulomatosis

$p-A N C A$

- commonest antigen is myeloperoxidase

- associated with polyarteritis nodosa, Churg Strauss syndrome and idiopathic crescentic glomerulonephritis, in addition to Wegener's granulomatosis

Box 2

\section{ACR criteria for Wegener's granulomatosis}

Two or more of:

- abnormal urine sediment (rbc casts $/>5 \mathrm{rbc}$ )

- abnormal chest X-ray (nodules/cavities/fixed infiltrates)

- oral ulcers/nasal discharge

- granulomatosis inflammation on biopsy

Box 3

largest controlled trial yet completed supported its use in those patients who were dialysis-dependent at presentation. ${ }^{4}$ The ongoing collaborative European study should verify the indications for plasmapheresis.

This patient was treated with plasmapheresis, in addition to oral prednisolone and cyclophosphamide. She made a good recovery, becoming dialysis-independent after 10 days and audiometry confirmed recovery of hearing to close to normal. CT confirmed resolution of the inflammatory mass affecting the auditory apparatus.

\section{Final diagnosis}

Wegener's granulomatosis.

Keywords: ANCA, Wegener's granulomatosis

3 Robinson AJ. ANCA and the systemic necrotizin vasculitides. Nephrol Dial Transplant 1994; 9: 119-26. 4 Pusey CD, Ress AJ, Evans DJ, Peters DK, Lockwood CM. Plasma exchange in focal necrotizing glomerulonephritis without anti-GBM antibodies. Kidney Int 1991; 40: 757 63. 